\title{
Incompressible Rayleigh-Taylor mixing in circular and spherical geometries
}

\author{
G. Boffetta $\odot$ and S. Musacchio \\ Department of Physics and INFN, University of Torino, via P. Giuria 1, 10125 Torino, Italy
}

(Received 1 August 2021; revised 13 January 2022; accepted 29 January 2022; published 14 February 2022)

\begin{abstract}
We present a numerical investigation of the turbulent evolution of the mixing layer developing from the Rayleigh-Taylor instability for miscible incompressible fluids in circular (in two dimensions) and in spherical (in three dimensions) geometries in the Boussinesq approximation. We show that the main difference caused by the convergent geometry with respect to the planar case is that the center of the mixing layer drifts toward the center of the domain during the evolution of the mixing layer. A similar effect is observed for the radial profile of the density flux. We derive a simple geometrical relation for this inward drift based on mass conservation. In the late stage of the evolution we observe also the appearance of an inward-outward asymmetry in the radial profiles.
\end{abstract}

DOI: 10.1103/PhysRevE.105.025104

\section{INTRODUCTION}

Rayleigh-Taylor (RT) instability and mixing, produced by the relative acceleration of two fluids of different densities, is of great importance in many natural and industrial processes. RT turbulent mixing occurs in disciplines as diverse as astrophysics [1,2], atmospheric physics [3], inertial confinement fusion [4], and laser-matter interactions [5,6] (see also [7-11] for recent reviews).

In many of these applications, one needs to take into account different physical effects due to, e.g., nonBoussinesq effects, compressibility, and immiscibility (see, e.g., Refs. [12-16]). Nonetheless, even in these cases, the incompressible Boussinesq model is often used for its theoretical and numerical simplicity [17].

Most of the studies in RT instability and mixing are performed in a flat geometry, with a planar interface orthogonal to the uniform acceleration. Nonetheless, early studies already discussed also the RT instability in spherical or cylindrical geometry, in particular for application to cavitation [18-21]. More recently, linear and weakly nonlinear analysis of RT phenomena in cylindrical and spherical geometry with a radial acceleration has been done by several authors and the effects of convergent and divergent geometry on the linear instability and on the weakly nonlinear evolution have been discussed [22-25].

In this work we investigate, by means of direct numerical simulations of the Boussinesq model, the effects of the interface curvature on the turbulent, strongly nonlinear stage of the evolution of the mixing layer between two miscible, incompressible fluids in two-dimensional (2D) circular and three-dimensional (3D) spherical geometries. It is well known that dimensionality of the flow affects the dynamics of the RT system [26]. In 3D the buoyancy force is mostly active at large scale and it originates a direct cascade of kinetic energy inside the mixing layer with Kolmogorov-Obukhov scaling laws for the velocity and density fluctuations [26,27]. In 2D the buoyancy is balanced scale by scale by the process of the inverse energy cascade. This results in the Bolgiano-Obukhov phenomenology [26,28]. A dynamical transition from 3D to 2D scaling has been observed in numerical simulations of the RT system confined in a thin layer [29]. The different scaling of the RT system in 3D and 2D has motivated a strong interest for the $2 \mathrm{D}$ case $[28,30]$, while the role of dimensionality in RT systems has been addressed in general in recent reviews $[7,9]$. Moreover, dimensional effects on the mixing properties in both bulk and porous RT has been recently investigated in [31].

Here, we focus on the influences of the convergent geometry, which are expected to depend on the dimensionality of the flow as well. We find that several properties remain almost unaffected with respect to the planar case. The shape of the density profile is very well captured by a nonlinear diffusive model developed for planar RT [32]. The temporal growth of the width of the mixing layer follows the dimensional prediction $h(t) \propto t^{2}$ with a dimensionless coefficient close to that of the planar case. Also the scaling of the Nusselt number as a function of the Rayleigh number is unchanged.

The main effect of the circular and spherical geometry is an inward drift of the central radial position of the density profile which appears during the expansion of the mixing layer. We show that this effect is due to the conservation of mass and it can be predicted in terms of a simple geometrical relation between the position of the center and the extension of the mixing layer. The explicit form of the geometrical relation is different in 2D circular and 3D spherical geometry, thus confirming the importance of the dimensionality in the evolution of the system. The profile of density flux displays a similar drift and, moreover, it develops an inward-outward asymmetry in the late stage of the evolution.

\section{MODELS AND METHODS}

We consider the Boussinesq model for an incompressible velocity field $\boldsymbol{u}(\boldsymbol{r}, t)$ coupled to a density field 
$\rho(\boldsymbol{r}, t)=\rho_{0}[1+\theta(\boldsymbol{r}, t)]$ in a circular and spherical domain (of radius $R_{1}$ )

$$
\begin{gathered}
\frac{\partial \boldsymbol{u}}{\partial t}+\boldsymbol{u} \cdot \nabla \boldsymbol{u}=-\frac{1}{\rho_{0}} \nabla p+v \nabla^{2} \boldsymbol{u}+\boldsymbol{g} \theta \\
\frac{\partial \theta}{\partial t}+\boldsymbol{u} \cdot \nabla \theta=\kappa \nabla^{2} \theta
\end{gathered}
$$

with the acceleration acting in the radial direction $\hat{\boldsymbol{r}}, \boldsymbol{g}=g \hat{\boldsymbol{r}}$. We remark that, within the Boussinesq approximation, the direction of $\boldsymbol{g}$ (i.e., the sign of the amplitude $g$ ) is irrelevant since Eqs. (1) and (2) are invariant as $\boldsymbol{g} \rightarrow-\boldsymbol{g}$ and $\theta \rightarrow-\theta$. Therefore, the dynamics in the convergent and divergent geometry are equivalent and in the following we will consider the case $g>0$ only. The initial unstable density profile we take is

$$
\theta(\boldsymbol{r}, 0)=\left(\theta_{0} / 2\right) \operatorname{sgn}\left(R_{0}-r\right)
$$

where $R_{0}<R_{1}$ is the initial position of the unstable interface and $\theta_{0}=\Delta \rho / \rho_{0}$ represents the dimensionless density jump which defines the Atwood number $A=\theta_{0} / 2$. The density of the inner fluid (for $r<R_{0}$ ) is $\rho_{\text {in }}=\rho_{0}\left(1+\theta_{0} / 2\right)$, while the outer fluid (for $r>R_{0}$ ) has density $\rho_{\text {out }}=\rho_{0}\left(1-\theta_{0} / 2\right)$. We remark that the Boussinesq model (1) and (2) is obtained under the assumption of small Atwood numbers and, within this limit, the value of $A$ simply rescales the characteristic time of the evolution.

Linear stability analysis of cylindrical [33] and spherical [25] interfaces shows that, for sufficiently small viscosity, the most unstable mode $k_{\max }$ is at short wavelength (i.e., $k_{\max } R_{0} \gg 1$ ) and therefore the curvature of the interface can be neglected and the growth rate for wave number $k$ can be approximated by $\gamma(k)=\sqrt{A g k+\left(\nu k^{2}\right)^{2}}-v k^{2}$ [34]. As the instability enters into the nonlinear phase, and eventually becomes turbulent, the typical scale of the perturbation (the size of the density plumes) increases [7] and curvature effects are expected to become relevant.

In order to study the effects of the circular domain in the evolution of RT turbulence, we performed a set of direct numerical simulations of Eqs. (1) and (2) with a pseudospectral code. Simulations are done in a square domain of size $\mathcal{L}$ on a regular Cartesian grid at resolution $N^{2}=2048^{2}$. We have checked that the effect of the grid geometry is irrelevant by comparing the results with simulations at resolution $N^{2}=4096^{2}$. Polar singularity is avoided by putting $g=0$ in the origin and the gravity field $g \hat{\mathbf{r}}$ is applied in the domain $r \leqslant R_{1}=2 R_{0}$. Simulations are stopped when density fluctuations exit this domain. The perturbation on the circular interface at $r=R_{0}$ is given by a superposition of modes around $k_{\max }$ with random phases. $M=10$ simulations with different realizations of the random phases are averaged in order to increase the statistical accuracy of the results. While most of the results presented are in two dimensions, we have also performed one simulation of the spherical 3D geometry at moderate resolution $N^{3}=512^{3}$ to compare the effects of the dimensionality.

Results will be made dimensionless by using $R_{0}$ and $\tau=\left(R_{0} / A g\right)^{1 / 2}$ as space and time units, respectively. The parameters of the simulations are $A g=0.25, v=\kappa=10^{-4}$
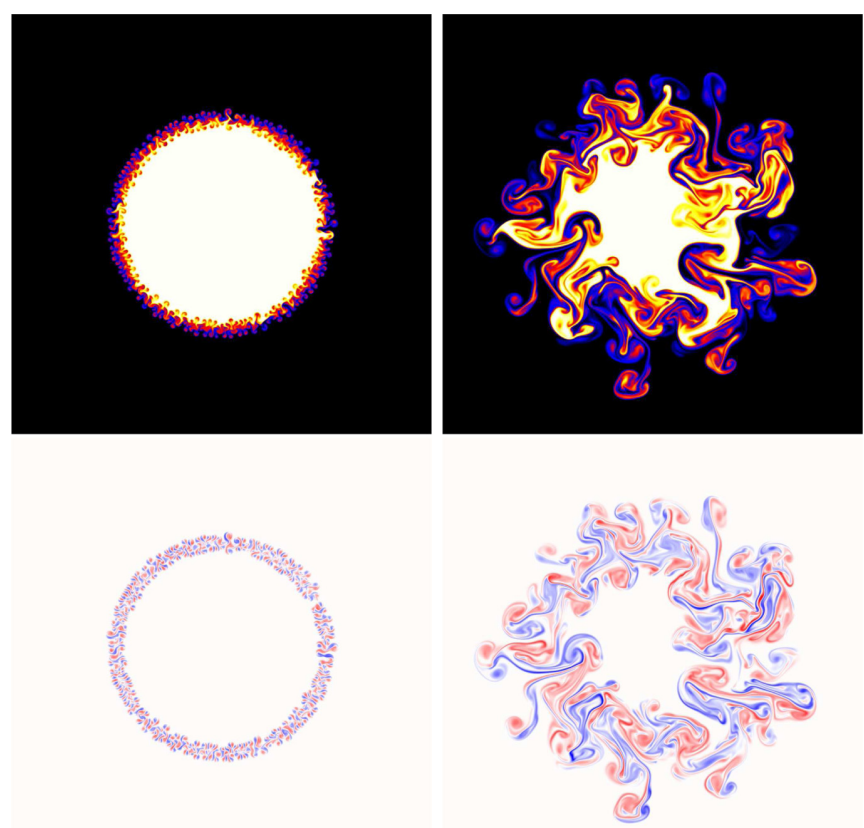

FIG. 1. Snapshots of the density field (upper) and of the vorticity field (lower) at an early time ( $t=1.6 \tau$, left) and in the late stage of the evolution ( $t=4.8 \tau$, right) from a two-dimensional simulation. Colors represent different densities, from $\theta=0.5 \theta_{0}$ (white) to $\theta=$ $-0.5 \theta_{0}$ (black) and vorticity (blue and red for positive and negative)

( $v=\kappa=5 \times 10^{-4}$ in $3 \mathrm{D}$ ), $\mathcal{L}=2 \pi$, and $R_{0}=\mathcal{L} / 4$. In the analysis of the numerical data we will consider radial profiles defined as the average of the angular coordinate (and over the different realizations), as for the radial density profile $\bar{\theta}(r)=\frac{1}{S_{d}} \int \theta\left(\boldsymbol{r}^{\prime}\right) \delta\left(\left|\boldsymbol{r}^{\prime}\right|-r\right) d^{d} \boldsymbol{r}^{\prime}$, where $S_{d}(r)$ is the surface of the $d$ sphere of radius $r$.

\section{RESULTS}

The development of the RT instability produces a mixing layer which expands radially both in the inner and outer directions. Figure 1 shows two snapshots of the density field and of the vorticity field in the early and late stage of the evolution. It is evident that, while the typical size of the initial plumes is much smaller than the radius of interface $R_{0}$, at later times they have grown to a size comparable with the radius of the internal interface and therefore we expect that curvature effects become important and that the inner-outer symmetry around $R_{0}$ is broken.

In Fig. 2 we plot the radial density profile $\bar{\theta}(r)$ at time $t=4 \tau$ together with the initial jump profile at $t=0$. As in planar RT turbulence the mixing region develops an almost linear density profile which connects the two unmixed regions. The extension of the mixing layer $h(t)$, defined as the region where $|\bar{\theta}(r)|<\theta_{0} / 2$, is usually defined in terms of a local threshold value [35] or integral quantities [2]. Here we use a nonlinear diffusive model, recently developed for planar RT turbulence [32], that fits the numerical data remarkably well. The model, based on a density dependent diffusivity, 


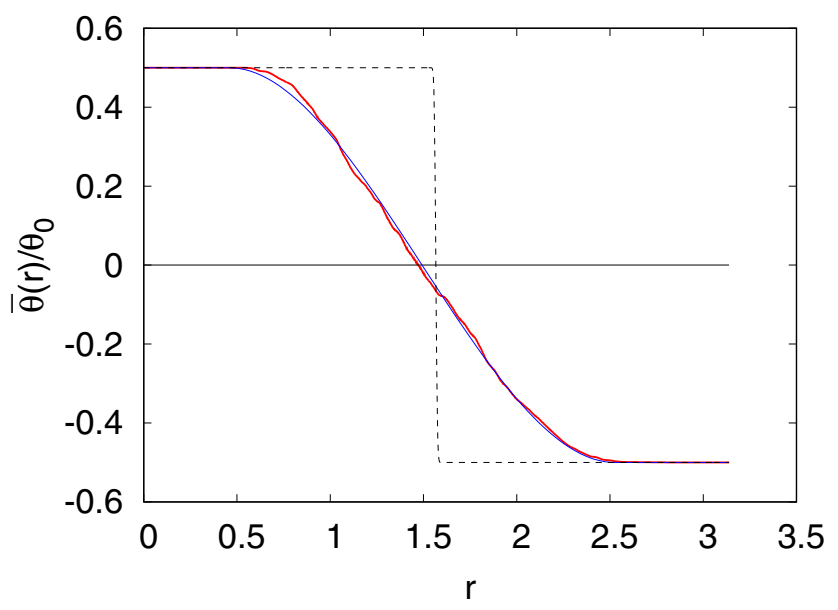

FIG. 2. Radial density profile $\bar{\theta}(r)$ averaged over 10 independent runs of the $2 \mathrm{D}$ simulation at time $t=4.0 \tau$ (red continuous line). Black dashed line represents the initial profile at $t=0$. Blue dotted line is the fit with the diffusive model (4).

predicts for the mixing layer the polynomial profile

$$
\bar{\theta}(r)=\theta_{0} \frac{r-R_{c}}{4 L}\left(\frac{\left(r-R_{c}\right)^{2}}{L^{2}}-3\right)
$$

centered on $R_{c}$ and defined for $R_{c}-L \leqslant r \leqslant R_{c}+L$ [while $\bar{\theta}(r)=\theta_{0} / 2$ for $r \leqslant R_{c}-L$ and $\bar{\theta}(r)=-\theta_{0} / 2$ for $r \geqslant R_{c}+$ $L]$. Within this model, the values of $L$ and $R_{c}$ are free parameters which are determined by fitting the radial density profiles obtained from the numerical simulations. In the current study, the width of the mixing layer is defined as $h=2 L$ from the values of the parameter $L$ given by the fit.

We remark that the profile (4) is antisymmetric with respect to the central point $R_{c}$ at which $\bar{\theta}=0$. While this is justified in the case of the planar Boussinesq model, in this case we observe a small departure from the inward-outward symmetry in the late stage of the evolution. Nonetheless, as shown in Fig. 2, the model (4) is able to reproduce accurately the radial density profile and to measure the extension of the mixing layer both in 2D and in 3D.

The efficiency of the mixing process can be characterized by the mixed mass [36]. Within the Boussinesq approximation the mixed mass is proportional to the variance of the density field and, for the present configuration, can be written as

$$
\mathcal{M}=\int S_{d}(r) m(r) d r,
$$

where $m(r)=\left[\theta_{0}^{2}-4 \overline{\theta^{2}}(r)\right] / \theta_{0}^{2}$ is the mixed mass profile which varies between 0 and 1 . Figure 3 shows the mixed mass profiles at the three different times (two corresponding to Fig. 1). We observe that while at early time the mixing is almost complete (because the small scales of the density plumes), at later times the mixed mass profile reaches a constant value $m \simeq 0.6$ in the center of the mixing layer. At later times we also observe a shift of the profile towards the center of the domain. The time evolution of the global mixed mass is plotted in the inset of Fig. 4.

Figure 4 plots the width of the mixing layer $h(t)$ obtained by fitting the density profiles at different times with

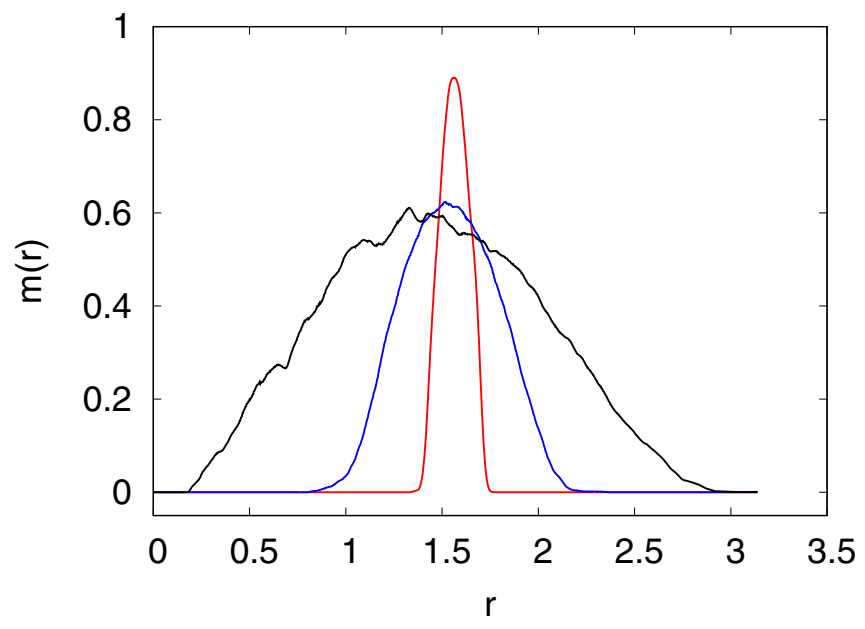

FIG. 3. Mixed mass profile $m(r)$ averaged over 10 independent runs of the $2 \mathrm{D}$ simulations at time $t=1.6 \tau$ (red line), $t=3.2 \tau$ (blue line), and $t=4.8 \tau$ (black line).

the model (4). At short time, the motion of coherent density plumes produces an overshooting in the extension of the mixing layer, while for $t \geqslant 2 \tau$ the incoherent and turbulent mixing layer grows according to the dimensional prediction $h(t) \simeq \alpha A g t^{2}$ (see compensated plot in the inset of Fig. 4). The precise value of the dimensionless coefficient $\alpha=0.084$ is obtained by fitting $h(t)$ with the quadratic law of the type $h(t)=\alpha A g\left(t-t_{0}\right)^{2}$, which takes into account the presence of the initial transient [37]. This result is compatible with 2D simulations in planar configuration [31] (taking into account the different definitions of $h$ ).

From Fig. 2 (and also from Fig. 3) it is evident that the zero-crossing point $R_{c}(t)$ at long time is different from $R_{0}$.

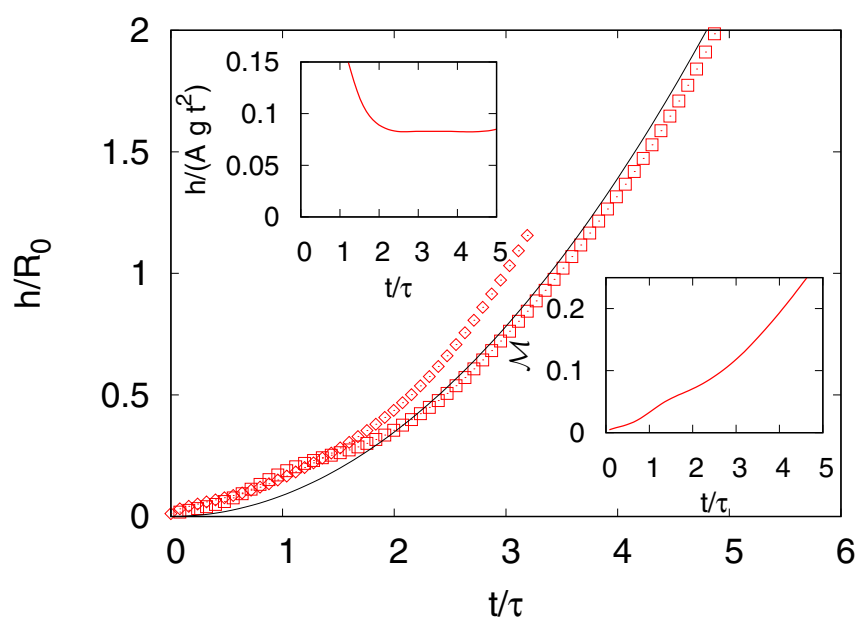

FIG. 4. Temporal growth of the mixing layer $h(t)=2 L$ computed from the fit of the 2D simulations with the model (4) at different times. The black line represents the dimensional prediction $h(t)=\alpha A g\left(t-t_{0}\right)^{2}$ fitted for $t \geqslant 2 \tau$ with $\alpha=0.084$. Red diamonds represent the evolution for the 3D simulation. Upper inset: $h(t)$ compensated with the dimensional prediction $A g t^{2}$. Lower inset: mixed mass $\mathcal{M}$ as a function of time. 


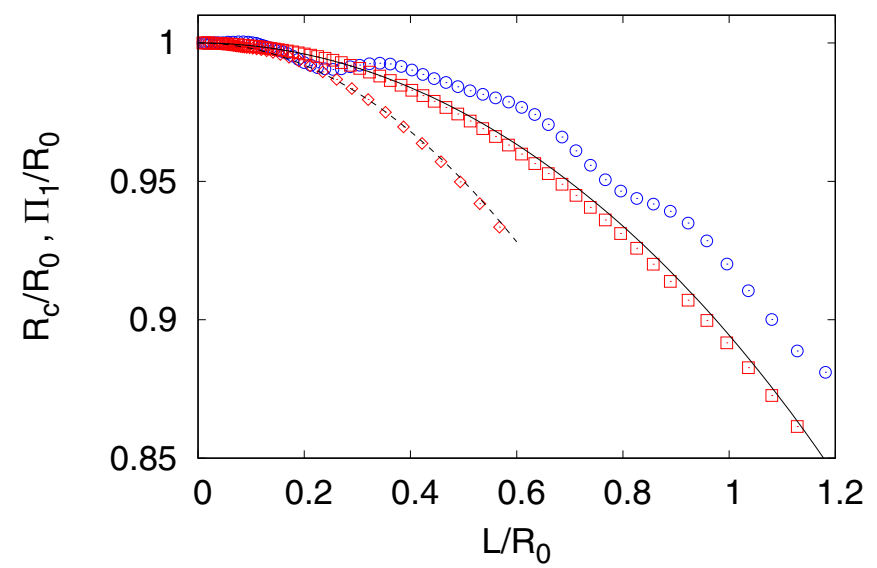

FIG. 5. Relative displacement of the central position of the profile $R_{c}$ (red squares) and position of the density flux $\Pi_{1}$ (blue circles) as a function of the half mixing layer width $L . R_{c}$ is computed as the point at which $\bar{\theta}\left(R_{c}\right)=0$. Red diamonds represent the central position $R_{c}$ from 3D simulation. The black continuous line represents the geometrical prediction (6) in $2 \mathrm{D}$; the black dashed line is the $3 \mathrm{D}$ prediction (7).

This is a consequence of mass conservation. In two dimensions, the mass of the system of two fluids is given by $M(t)=$ $\int \bar{\rho}(r, t) 2 \pi r d r=M_{\text {out }}+\int \rho_{0}\left[\bar{\theta}(r, t)+\theta_{0} / 2\right] 2 \pi r d r$, where $M_{\text {out }}=\int \rho_{\text {out }} 2 \pi r d r$ is the mass of the whole volume filled with the fluid with smaller density $\rho_{\text {out }}$, while the integral $\int \rho_{0}\left[\bar{\theta}(r, t)+\theta_{0} / 2\right] 2 \pi r d r$ is the extra mass due to the heavier fluid with density $\rho_{\text {in }}=\rho_{\text {out }}+\Delta \rho$ (with $\Delta \rho=\rho_{0} \theta_{0}$ ). At the initial time $t=0$ one has $M(0)=M_{\text {out }}+\rho_{0} \theta_{0} \pi R_{0}^{2}$. The mass at time $t$ can be computed using the expression (4) for the density profile. Imposing the conservation of mass $M(t)=$ $M(0)$ one obtains the simple geometrical relation $5 R_{c}^{2}+L^{2}=$ $5 R_{0}^{2}$ and therefore a relation between the central point $R_{c}$ and the extension of the mixing layer $L$

$$
\frac{R_{c}}{R_{0}}=\sqrt{1-\frac{L^{2}}{5 R_{0}^{2}}} .
$$

Relation (6) shows that $R_{c}(t) \leqslant R_{0}$, i.e., the interface moves toward the center of the domain as the mixing layer grows. We remark that the quadratic dependence in (6) is general since it is a consequence of mass preservation in $2 \mathrm{D}$, while the numerical coefficients depend on the specific model for $\bar{\theta}(r)$. Recently, a model for the initial stage of instability, in the weakly nonlinear regime, has been proposed on the basis of a similar principle of mass conservation [23].

The geometrical relation (6) can be generalized to arbitrary dimensions by equating the initial mass $M(0)=\int \bar{\rho}(r, 0) d^{d} r$ with the mass $M(t)=\int \bar{\rho}(r, t) d^{d} r$ at time $t$. In general one obtains a relation of the form $R_{0}^{d}=\left(R_{c}+L\right)^{d} f_{+}\left(R_{c} / L\right)+$ $\left(R_{c}-L\right)^{d} f_{-}\left(R_{c} / L\right)$, in which the explicit form of the functions $f_{ \pm}$depends on the dimension $d$ and on the functional form of the radial density profile. In three dimensions, using the expression (4) one obtains the simple relation

$$
5 R_{c}^{3}+3 L^{2} R_{c}=5 R_{0}^{3}
$$

which gives the dependence of $R_{c}$ as a function of $L$.

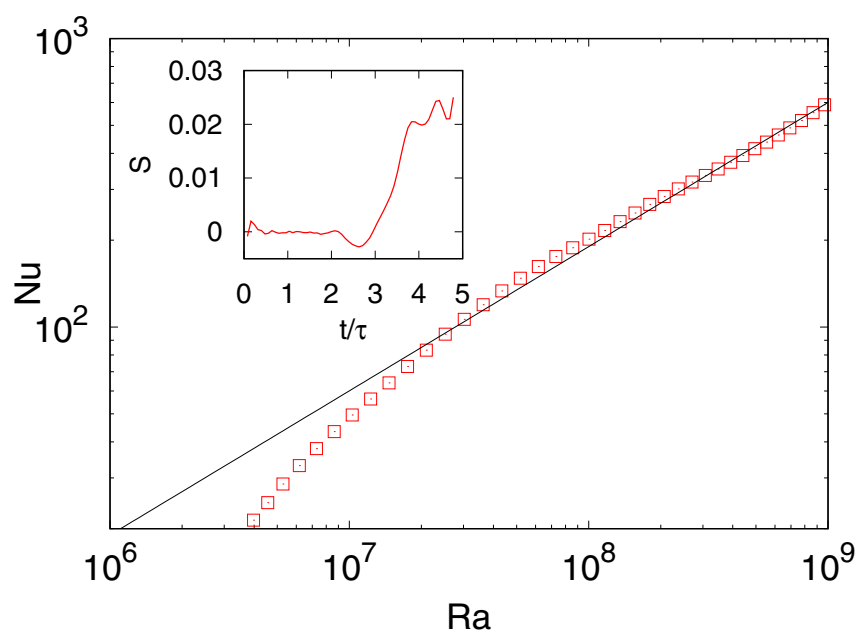

FIG. 6. Nusselt number as a function of the Rayleigh number. The black continuous line represents the ultimate scaling $\mathrm{Nu}=$ $0.02 \mathrm{Ra}$. Inset: skewness of the density flux $S$ as a function of time.

In Fig. 5 we plot the relation between $R_{c}$ and $L$ obtained from numerical simulations together with the geometrical predictions (6) and (7). In 2D, we observe that at the end of the simulation the center of the mixing layer has moved about $15 \%$ towards the center of the domain. In $3 \mathrm{D}$ we find that this geometrical effect is enhanced, because of the different dependence of the volume element on the radial coordinate $r$. Both in 2D and 3D, the geometrical models (6) and (7) are in excellent agreement with the numerical data, without free parameters. We remark that when $R_{c} \leqslant L$ the mixing layer has completely filled the inner part of the interface. Nonetheless, we find that, even in this case, the model (4) fits the density profile well and (6) is in agreement with the data.

We also investigate the effects of the circular geometry on the radial density flux $\left\langle u_{r} \theta\right\rangle \equiv \frac{1}{2 L} \int_{R_{c}-L}^{R_{c}+L} \pi(r) d r$, which is defined as the average over the extension of the mixing layer of the radial profile of density flux $\pi(r)=\overline{u_{r} \theta}(r)$, where $u_{r}$ is the radial component of the velocity field.

The density flux is quantified in dimensionless variables by the Nusselt number $\mathrm{Nu}=1+\left\langle u_{r} \theta\right\rangle h /\left(\kappa \theta_{0}\right)$. Extensive numerical simulations have shown that in the Rayleigh-Taylor mixing the Nusselt number scaling, as a function of the Rayleigh number $\mathrm{Ra}=g \theta_{0} h^{3} /(\nu \kappa)$, follows the ultimate state regime $\mathrm{Nu} \sim \mathrm{Ra}^{1 / 2}$ [38]. Figure 6 shows that the ultimate state scaling is observed also in the circular geometry in the late stage of the simulation, when $\mathrm{Ra} \geqslant 10^{8}$. We find a close agreement with the results of numerical simulations in $2 \mathrm{D}$ planar geometry [28].

Similar to what is observed for the density profile, the main effect of the circular geometry on the density flux is an inward drift of its mean radial position. This effect can be quantified by introducing the moments of the radial flux $\Pi_{p}=\int r^{p} \pi(r) d r / \int \pi(r) d r$. The mean radial position of the density flux is given by $\Pi_{1}$. As shown in Fig. $5, \Pi_{1}$ moves towards the center of the circular domain as the width of the mixing layer increases. The inward drift of the mean radial position of the flux $\Pi_{1}$ is similar to what is observed for the center of the density profile $R_{c}$. 
A measure of the asymmetry of the radial profile of density flux $\pi(r)$ is provided by the skewness $S=\left(\Pi_{3}-\Pi_{1}^{3}\right) /\left(\Pi_{2}-\right.$ $\left.\Pi_{1}^{2}\right)^{3 / 2}$. As shown in the inset of Fig. 6, we find that in the early stage of the evolution $t \leqslant 2 \tau$ the skewness is almost null and therefore the flux is symmetric, as in the planar RT [38]. The inward-outward symmetry of the flux breaks suddenly at $t \simeq 3 \tau$, when the skewness becomes positive indicating that in the late stage of the evolution the density flux is more intense toward the outer region of the mixing layer. We note that this asymmetry is not trivially related to the inward drift of $\Pi_{1}$, which manifests already at the beginning of the evolution.

\section{CONCLUSIONS}

We have studied the effects of the curvature of the interface in incompressible Rayleigh-Taylor turbulent mixing in a circular 2D and spherical 3D domains subject to radial acceleration. The main result of our study is that, while the shape of the density profile remains almost unaffected with respect to the planar interface, its central radial position $R_{c}$ moves toward the center of the domain as a consequence of the mass conservation in the convergent geometry. We have shown that in 2D the inward drift of $R_{c}$ is well captured by a geometrical relation without free parameters which involves solely the initial radial position of the interface $R_{0}$ and the width of the mixing layer $L$. The simulation in $3 \mathrm{D}$ spherical geometry shows that this geometrical effect is stronger than in $2 \mathrm{D}$, and that it can be predicted by the same physical argument. It would be interesting to extend the study of the effects of convergent geometry also to cases in which the radial acceleration $g$ is not constant and/or to more realistic models beyond the Boussinesq approximation.
[1] M. Zingale, S. E. Woosley, C. A. Rendleman, M. S. Day, and J. B. Bell, Three-dimensional numerical simulations of Rayleigh-Taylor unstable flames in type Ia supernovae, Astrophys. J. 632, 1021 (2005).

[2] W. H. Cabot and A. W. Cook, Reynolds number effects on Rayleigh-Taylor instability with possible implications for type Ia supernovae, Nat. Phys. 2, 562 (2006).

[3] M. C. Kelley, G. Haerendel, H. Kappler, A. Valenzuela, B. B. Balsley, D. A. Carter, W. L. Ecklund, C. W. Carlson, B. Häusler, and R. Torbert, Evidence for a Rayleigh-Taylor type instability and upwelling of depleted density regions during equatorial spread F, Geophys. Res. Lett. 3, 448 (1976).

[4] S. Atzeni and J. Meyer-ter Vehn, The Physics of Inertial Fusion: Beam Plasma Interaction, Hydrodynamics, Hot Dense Matter (Oxford University Press, Oxford, 2004), Vol. 125.

[5] A. Sgattoni, S. Sinigardi, L. Fedeli, F. Pegoraro, and A. Macchi, Laser-driven Rayleigh-Taylor instability: Plasmonic effects and three-dimensional structures, Phys. Rev. E 91, 013106 (2015).

[6] K. Shigemori, H. Azechi, M. Nakai, M. Honda, K. Meguro, N. Miyanaga, H. Takabe, and K. Mima, Measurements of Rayleigh-Taylor Growth Rate of Planar Targets Irradiated Directly by Partially Coherent Light, Phys. Rev. Lett. 78, 250 (1997).

[7] G. Boffetta and A. Mazzino, Incompressible Rayleigh-Taylor turbulence, Annu. Rev. Fluid Mech. 49, 119 (2017).

[8] Y. Zhou, Rayleigh-Taylor and Richtmyer-Meshkov instability induced flow, turbulence, and mixing. I, Phys. Rep. 720-722, 1 (2017).

[9] Y. Zhou, T. T. Clark, D. S. Clark, S. G. Glendinning, M. A. Skinner, C. M. Huntington, O. A. Hurricane, A. M. Dimits, and B. A. Remington, Turbulent mixing and transition criteria of flows induced by hydrodynamic instabilities, Phys. Plasmas 26, 080901 (2019).

[10] A. Banerjee, Rayleigh-Taylor Instability: A Status Review of Experimental Designs and Measurement Diagnostics, J. Fluids Eng. 142, 120801 (2020).

[11] O. Schilling, Progress on understanding Rayleigh-Taylor flow and mixing using synergy between simulation, modeling, and experiment, J. Fluids Eng. 142, 120802 (2020).
[12] D. Livescu, Compressibility effects on the Rayleigh-Taylor instability growth between immiscible fluids, Phys. Fluids 16, 118 (2004).

[13] A. Celani, A. Mazzino, P. Muratore-Ginanneschi, and L. Vozella, Phase-field model for the Rayleigh-Taylor instability of immiscible fluids, J. Fluid Mech. 622, 115 (2009).

[14] D. Livescu, J. R. Ristorcelli, R. A. Gore, S. H. Dean, W. H. Cabot, and A. W. Cook, High-Reynolds number RayleighTaylor turbulence, J. Turbulence 10, N13 (2009).

[15] D. Aslangil, D. Livescu, and A. Banerjee, Effects of Atwood and Reynolds numbers on the evolution of buoyancy-driven homogeneous variable-density turbulence, J. Fluid Mech. 895, A12 (2020).

[16] D. Aslangil, D. Livescu, and A. Banerjee, Variable-density buoyancy-driven turbulence with asymmetric initial density distribution, Physica D 406, 132444 (2020).

[17] Y. Zhou, R. J. R. Williams, P. Ramaprabhu, M. Groom, B. Thornber, A. Hillier, W. Mostert, B. Rollin, S. Balachandar, P. D. Powell et al., Rayleigh-Taylor and Richtmyer-Meshkov instabilities: A journey through scales, Physica D 423, 132838 (2021).

[18] A. M. Binnie, The stability of the surface of a cavitation bubble, Math. Proc. Cambridge Philos. Soc. 49, 151 (1953).

[19] S. Chandrasekhar, The character of the equilibrium of an incompressible heavy viscous fluid of variable density, Math. Proc. Cambridge Philos. Soc. 51, 162 (1955).

[20] M. S. Plesset and A. Prosperetti, Bubble dynamics and cavitation, Annu. Rev. Fluid Mech. 9, 145 (1977).

[21] H. J. Kull, Theory of the Rayleigh-Taylor instability, Phys. Rep. 206, 197 (1991).

[22] K. O. Mikaelian, Stability and Mix in Spherical Geometry, Phys. Rev. Lett. 65, 992 (1990).

[23] L. F. Wang, J. F. Wu, W. H. Ye, W. Y. Zhang, and X. T. He, Weakly nonlinear incompressible Rayleigh-Taylor instability growth at cylindrically convergent interfaces, Phys. Plasmas 20, 042708 (2013).

[24] G. Terrones and M. D. Carrara, Rayleigh-Taylor instability at spherical interfaces between viscous fluids: Fluid/vacuum interface, Phys. Fluids 27, 054105 (2015). 
[25] K. O. Mikaelian, Viscous Rayleigh-Taylor instability in spherical geometry, Phys. Rev. E 93, 023104 (2016).

[26] M. Chertkov, Phenomenology of Rayleigh-Taylor Turbulence, Phys. Rev. Lett. 91, 115001 (2003).

[27] G. Boffetta, A. Mazzino, S. Musacchio, and L. Vozella, Kolmogorov scaling and intermittency in Rayleigh-Taylor turbulence, Phys. Rev. E 79, 065301(R) (2009).

[28] A. Celani, A. Mazzino, and L. Vozella, Rayleigh-Taylor Turbulence in Two Dimensions, Phys. Rev. Lett. 96, 134504 (2006).

[29] G. Boffetta, F. De Lillo, and S. Musacchio, Bolgiano scale in confined Rayleigh-Taylor turbulence, J. Fluid Mech. 690, 426 (2012).

[30] L. Biferale, F. Mantovani, M. Sbragaglia, A. Scagliarini, F. Toschi, and R. Tripiccione, High resolution numerical study of Rayleigh-Taylor turbulence using a thermal lattice Boltzmann scheme, Phys. Fluids 22, 115112 (2010).

[31] G. Boffetta and S. Musacchio, Dimensional effects in RayleighTaylor mixing, Phil. Trans. R. Soc. A 380, 20210084 (2022).

[32] G. Boffetta, F. De Lillo, and S. Musacchio, Nonlinear Diffusion Model for Rayleigh-Taylor Mixing, Phys. Rev. Lett. 104, 034505 (2010).
[33] H. Yu and D. Livescu, Rayleigh-Taylor instability in cylindrical geometry with compressible fluids, Phys. Fluids 20, 104103 (2008).

[34] R. Menikoff, R. C. Mjolsness, D. H. Sharp, and C. Zemach, Unstable normal mode for Rayleigh-Taylor instability in viscous fluids, Phys. Fluids 20, 2000 (1977).

[35] S. B. Dalziel, P. F. Linden, and D. L. Youngs, Self-similarity and internal structure of turbulence induced by Rayleigh-Taylor instability, J. Fluid Mech. 399, 1 (1999).

[36] Y. Zhou, W. H. Cabot, and B. Thornber, Asymptotic behavior of the mixed mass in Rayleigh-Taylor and RichtmyerMeshkov instability induced flows, Phys. Plasmas 23, 052712 (2016).

[37] A. Banerjee, W. N. Kraft, and M. J. Andrews, Detailed measurements of a statistically steady Rayleigh-Taylor mixing layer from small to high Atwood numbers, J. Fluid Mech. 659, 127 (2010).

[38] G. Boffetta, A. Mazzino, S. Musacchio, and L. Vozella, Statistics of mixing in three-dimensional Rayleigh-Taylor turbulence at low Atwood number and Prandtl number one, Phys. Fluids 22, 035109 (2010). 